\title{
Observation of cross-shaped anisotropy in spin-resolved small-angle neutron scattering
}

\author{
Andreas Michels, ${ }^{*}$ Dirk Honecker, and Frank Döbrich \\ Laboratory for the Physics of Advanced Materials, University of Luxembourg, 162A Avenue de la Faïencerie, \\ L-1511 Luxembourg, Luxembourg \\ Charles D. Dewhurst \\ Institut Laue-Langevin, 6 Rue Jules Horowitz, B.P. 156, F-38042 Grenoble Cedex 9, France \\ Kiyonori Suzuki \\ Department of Materials Engineering, Monash University, Clayton, Victoria 3800, Australia \\ André Heinemann \\ Institute of Materials Research, Helmholtz Zentrum Geesthacht, Max-Planck-Straße 1, D-21502 Geesthacht, Germany
}

(Received 22 March 2012; published 18 May 2012)

\begin{abstract}
We report the results of spin-resolved small-angle neutron scattering (SANS) experiments on the two-phase nanocrystalline alloy NANOPERM. At a saturating applied magnetic field of $1.27 \mathrm{~T}$ we observe a cross-shaped angular anisotropy in the non-spin-flip SANS cross section $\Sigma^{++}$. This feature-for this class of materials only visible at saturation in $\Sigma^{++}$-is attributed to the specific ratio of nuclear to magnetic scattering being smaller than unity. Analysis of the non-spin-flip and spin-flip cross sections provides the nuclear and magnetic SANS and allows us to estimate the magnitude of the respective scattering-length density contrast at the interphase between the nanoparticles and the amorphous magnetic matrix.
\end{abstract}

DOI: 10.1103/PhysRevB.85.184417

PACS number(s): 61.05.fg, 75.25.-j, 75.75.-c

\section{INTRODUCTION}

Soft magnetic nanocrystalline alloys such as FINEMET, NANOPERM, or HITPERM are the subject of intense fundamental and applied scientific research. ${ }^{1-3}$ Due to their extreme magnetic softness (coercivity values range between 1 and $50 \mathrm{~A} / \mathrm{m})$, large saturation polarization $(\sim 1.2-1.8 \mathrm{~T})$ and high initial permeability $\left(\sim 10^{3}-10^{5}\right)$, these materials are very attractive for modern electronics applications, e.g., as materials for transformer cores or chokes. ${ }^{4}$ Most of their relevant magnetic properties are "realized" on a microscopic length scale and are related to the specific microstructure, which consists of a dispersion of crystalline nanometer-sized magnetic particles that are embedded in an amorphous magnetic matrix. For instance, it is well known that the nanoscale particle size leads, via the mechanism of exchange softening, to the very low coercivity values, ${ }^{1}$ and that the specific composition profile (diffusion zone) around the precipitates is largely responsible for the transformation of the amorphous precursor alloy into the nanocrystalline state. ${ }^{5}$ In order to improve the performance of the nanocomposites, understanding of the microstructureproperty relationship is crucial. Therefore, it is important to have characterization techniques at one's disposal which are able to resolve the magnetic microstructure on the nanoscale and in the volume (bulk) of the material.

Small-angle neutron scattering (SANS) is such a method, which has previously demonstrated great potential for resolving the spin microstructures of soft magnetic nanocomposites. ${ }^{5-16}$ Here, we use magnetic SANS with one-dimensional (uniaxial) polarization analysis (denoted as POLARIS $)^{17-19}$ in order to investigate the magnetic microstructure of the two-phase Fe-based nanocrystalline alloy NANOPERM. Specifically, we focus in this report on the (nearly) saturated state, where the (usually dominating) magnetic SANS signal due to so-called spin-misalignment fluctuations is small and the magnetic scattering originates from nanoscale jumps of the magnetization at phase boundaries. In this situation, we observe an unusual cross-shaped angular anisotropy in the non-spin-flip SANS cross section.

\section{EXPERIMENT}

The neutron experiment was carried out at room temperature at the instrument D22 at the ILL, Grenoble, France. Incident neutrons with a wavelength of $\lambda=8 \AA$ and $\Delta \lambda / \lambda=10 \%$ (FWHM) were used. The beam was polarized by means of a 1.2-m-long remanent FeSi supermirror transmission polarizer $(m=3.6)$. A rf spin flipper, installed close to the sample position, allowed us to reverse the initial neutron polarization. Measurement of the four partial neutron scattering cross sections $\frac{d \Sigma^{++}}{d \Omega}, \frac{d \Sigma^{--}}{d \Omega}, \frac{d \Sigma^{+-}}{d \Omega}$, and $\frac{d \Sigma^{-+}}{d \Omega}$ was accomplished through a polarized ${ }^{3} \mathrm{He}$ spin-filter cell. The polarization between polarizer, rf flipper, and ${ }^{3} \mathrm{He}$ filter was maintained by means of magnetic guide fields of the order of $1 \mathrm{mT}$. The scattered neutrons were detected by a multitube detector which consists of $128 \times 128$ pixels with a resolution of $8 \times 8 \mathrm{~mm}$. Data reduction, including corrections for spin leakage, was performed using the GRASP and BERSANS ${ }^{20}$ software packages.

The sample under study was a magnetic nanocomposite from the NANOPERM family of alloys with a nominal composition of $\left(\mathrm{Fe}_{0.985} \mathrm{Co}_{0.015}\right)_{90} \mathrm{Zr}_{7} \mathrm{~B}_{3}$ (Ref. 21). Annealing of as-prepared melt-spun ribbons at a temperature of $610^{\circ} \mathrm{C}$ for $1 \mathrm{~h}$ resulted in a two-phase microstructure, which consists of a dispersion of bcc iron nanoparticles that are embedded in an amorphous magnetic matrix. Analysis of wide-angle X-ray diffraction data yielded a mean particle size of $D_{\mathrm{XRD}}=$ $(15 \pm 2) \mathrm{nm}$ and a root-mean-square microstrain of the order 
of $0.1 \%$. The crystalline volume fraction is about $x_{C} \cong 65 \%$, and the saturation polarization of the alloy amounts to $J_{s}=$ $1.64 \mathrm{~T}$. Addition of tiny amounts of Co results in a vanishing magnetostriction. ${ }^{21}$

\section{POLARIS CROSS SECTIONS}

In the SANS experiment, the applied magnetic (guide) field $\mathbf{H}$ was taken parallel to $\mathbf{e}_{z}$ (i.e., horizontally), and the wave vector $\mathbf{k}_{0}$ of the incident neutron beam was assumed to be parallel to $\mathbf{e}_{x}$. For this scattering geometry, the elastic differential POLARIS cross sections $d \Sigma / d \Omega$ can be expressed as $^{17-19}$

$$
\begin{aligned}
& \frac{d \Sigma^{ \pm \pm}}{d \Omega}(\mathbf{q})=\frac{8 \pi^{3}}{V}\left(|\tilde{N}|^{2}+b_{H}^{2}\left|\tilde{M}_{z}\right|^{2} \sin ^{4} \theta\right. \\
& +b_{H}^{2}\left|\tilde{M}_{y}\right|^{2} \sin ^{2} \theta \cos ^{2} \theta \\
& -b_{H}^{2}\left(\tilde{M}_{y} \tilde{M}_{z}^{*}+\tilde{M}_{y}^{*} \tilde{M}_{z}\right) \sin ^{3} \theta \cos \theta \\
& \mp b_{H}\left(\tilde{N} \tilde{M}_{z}^{*}+\tilde{N}^{*} \widetilde{M}_{z}\right) \sin ^{2} \theta \\
& \left. \pm b_{H}\left(\tilde{N} \tilde{M}_{y}^{*}+\tilde{N}^{*} \tilde{M}_{y}\right) \sin \theta \cos \theta\right) \text {, } \\
& \frac{d \Sigma^{ \pm \mp}}{d \Omega}(\mathbf{q})=\frac{8 \pi^{3}}{V} b_{H}^{2}\left(\left|\tilde{M}_{x}\right|^{2}+\left|\tilde{M}_{y}\right|^{2} \cos ^{4} \theta\right. \\
& +\left|\tilde{M}_{z}\right|^{2} \sin ^{2} \theta \cos ^{2} \theta \\
& \left.-\left(\tilde{M}_{y} \tilde{M}_{z}^{*}+\tilde{M}_{y}^{*} \tilde{M}_{z}\right) \sin \theta \cos ^{3} \theta\right),
\end{aligned}
$$

where $V$ is the sample volume, $\tilde{N}(\mathbf{q})$ and $\tilde{\mathbf{M}}(\mathbf{q})=$ $\left[\widetilde{M}_{x}(\mathbf{q}), \widetilde{M}_{y}(\mathbf{q}), \widetilde{M}_{z}(\mathbf{q})\right]$ denote, respectively, the Fourier coefficients of the nuclear scattering-length density and of the magnetization $\mathbf{M}(\mathbf{x})=\left[M_{x}(\mathbf{x}), M_{y}(\mathbf{x}), M_{z}(\mathbf{x})\right], b_{H}=$ $2.7 \times 10^{-15} \mathrm{~m} / \mu_{B}=2.9 \times 10^{8} \mathrm{~A}^{-1} \mathrm{~m}^{-1}$ (with $\mu_{B}$ the Bohr magneton), and $\theta$ represents the angle between $\mathbf{H}$ and the scattering vector $\mathbf{q} \cong q(0, \sin \theta, \cos \theta)$. Helical scattering terms have been neglected in Eq. (2). When the rf flipper is off, we measure, depending on the spin state of the ${ }^{3} \mathrm{He}$ filter, the non-spin-flip (nsf) or the spin-flip (sf) cross section $\frac{d \Sigma^{++}}{d \Omega}$ or $\frac{d \Sigma^{+-}}{d \Omega}$. Likewise, when the flipper is on, we either measure $\frac{d \Sigma^{--}}{d \Omega}$ or $\frac{d \Sigma^{-+}}{d \Omega}$. Furthermore, in Eqs. (1) and (2) (and in all other equations that follow), the polarization $P_{0}$ of the incident neutrons and the efficiency of the spin flipper were set to unity. Due to the neglect of chiral terms in Eq. (2), the two sf cross sections are independent of the sign of $P_{0}$, whereas the two nsf channels contain $P_{0}$-dependent terms.

At complete magnetic saturation, Eqs. (1) and (2) reduce to the simpler expressions

$$
\begin{aligned}
\frac{d \Sigma^{ \pm \pm}}{d \Omega}(\mathbf{q})= & \frac{8 \pi^{3}}{V}\left(|\tilde{N}|^{2}+b_{H}^{2}\left|\tilde{M}_{z}\right|^{2} \sin ^{4} \theta\right. \\
& \left.\mp b_{H}\left(\tilde{N} \tilde{M}_{z}^{*}+\tilde{N}^{*} \tilde{M}_{z}\right) \sin ^{2} \theta\right), \\
\frac{d \Sigma^{ \pm \mp}}{d \Omega}(\mathbf{q})= & \frac{8 \pi^{3}}{V} b_{H}^{2}\left|\tilde{M}_{z}\right|^{2} \sin ^{2} \theta \cos ^{2} \theta .
\end{aligned}
$$

As can be seen from Eq. (3), the relative magnitude of the nuclear $|\widetilde{N}|^{2}$ and magnetic $b_{H}^{2}\left|\widetilde{M}_{z}\right|^{2}$ SANS determines the angular anisotropy of $\frac{d \Sigma^{++}}{d \Omega}$. Figure 1 (upper row) shows the qualitative variation of $\frac{d \Sigma^{++}}{d \Omega}$ at magnetic saturation for different ratios of nuclear to magnetic scattering. In these computations we have assumed that both $\widetilde{N}$ and $\widetilde{M}_{z}$ are (a)

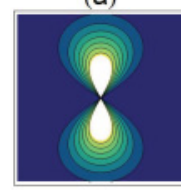

(e)

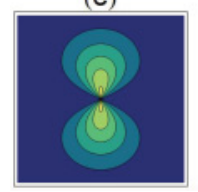

(b)

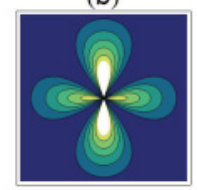

(f)

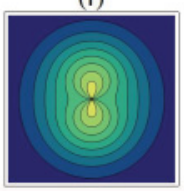

(c)

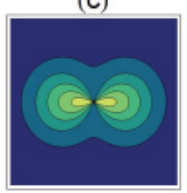

(g)

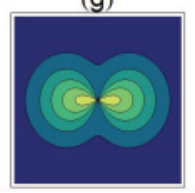

(d)

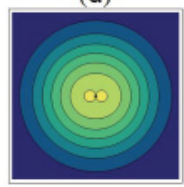

(h)

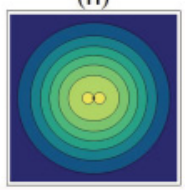

FIG. 1. (Color online) (upper row) Qualitative variation of $\frac{d \Sigma^{++}}{d \Omega}$ at magnetic saturation [Eq. (3)] as a function of the ratio $R$ of nuclear to magnetic scattering $\left[R:=|\widetilde{N}|^{2} /\left(b_{H}^{2}\left|\widetilde{M}_{z}\right|^{2}\right)\right]$. (a) $R=0.0025$, (b) $R=0.2025$, (c) $R=4.0$, (d) $R=2500$. For the calculation of $\frac{d \Sigma^{++}}{d \Omega}$ we have assumed the sphere form factor (sphere diameter: $16 \mathrm{~nm}$ ) for both $|\tilde{N}|^{2}$ and $\left|\widetilde{M}_{z}\right|^{2}$; the prefactor $8 \pi^{3} / V$ in Eq. (3) was set to unity. (lower row) (e)-(h) SANSPOL cross section $\frac{d \Sigma^{+}}{d \Omega}$ at saturation [Eq. (5)] and for the same $R$ values as in (a) $-(d)$, respectively.

positive-definite real-valued functions which depend only on the magnitude $q=|\mathbf{q}|$ of the scattering vector, as is the case for polycrystalline alloys of the NANOPERM type. ${ }^{15,22,23}$ For $|\tilde{N}|^{2} \ll b_{H}^{2}\left|\tilde{M}_{z}\right|^{2}$, the nsf ++ channel exhibits a dominating $\sin ^{4} \theta$ anisotropy [Fig. 1(a)], which changes to cross-shaped [Fig. 1(b)] and elliptically-shaped [Fig. 1(c)] for $|\tilde{N}|^{2} \cong b_{H}^{2}\left|\tilde{M}_{z}\right|^{2}$, and finally to isotropic for $|\tilde{N}|^{2} \gg b_{H}^{2}\left|\widetilde{M}_{z}\right|^{2}$ [Fig. 1(d)].

We would like to particularly emphasize that-at saturation-the cross-shaped anisotropy [Fig. 1(b)] is expected to be observable only in the ++ channel provided that the ratio $R$ of nuclear to magnetic scattering is smaller than unity. $^{24}$ This statement presupposes that the nuclear and magnetic scattering amplitudes $\widetilde{N}$ and $\widetilde{M}_{z}$ are positive-definite real-valued functions of $q$. If, for instance, $\widetilde{N}$ and $\widetilde{M}_{z}$ would possess opposite signs, then the cross-shaped anisotropy may appear (at saturation) in the -- channel. However, such and similar cases are not considered in the following discussion. The decisive point is that under the above specified conditions the cross-shaped anisotropy cannot be seen with unpolarized neutrons or with the so-called SANSPOL method, ${ }^{25}$ where the incident neutron beam is polarized but no uniaxial polarization analysis of the scattered neutrons is performed (compare lower row in Fig. 1). For SANSPOL and for $\mathbf{k}_{0} \perp \mathbf{H}$, the cross section at saturation reads

$$
\begin{aligned}
\frac{d \Sigma^{ \pm}}{d \Omega}(\mathbf{q})= & \frac{8 \pi^{3}}{V}\left(|\tilde{N}|^{2} \mp b_{H}\left(\tilde{N} \tilde{M}_{z}^{*}+\tilde{N}^{*} \tilde{M}_{z}\right) \sin ^{2} \theta\right. \\
& \left.+b_{H}^{2}\left|\tilde{M}_{z}\right|^{2} \sin ^{2} \theta\right)
\end{aligned}
$$

where + and - refer to the polarization state of the incident neutrons, in particular, $\frac{d \Sigma^{+}}{d \Omega}=\frac{d \Sigma^{++}}{d \Omega}+\frac{d \Sigma^{+-}}{d \Omega}$ and $\frac{d \Sigma^{-}}{d \Omega}=$ $\frac{d \Sigma^{--}}{d \Omega}+\frac{d \Sigma^{-+}}{d \Omega}$.

\section{RESULTS AND DISCUSSION}

Figure 2 displays the angular $(\theta)$ variation of the normalized intensity $I / I_{0}$ of the various spin-resolved cross sections at 

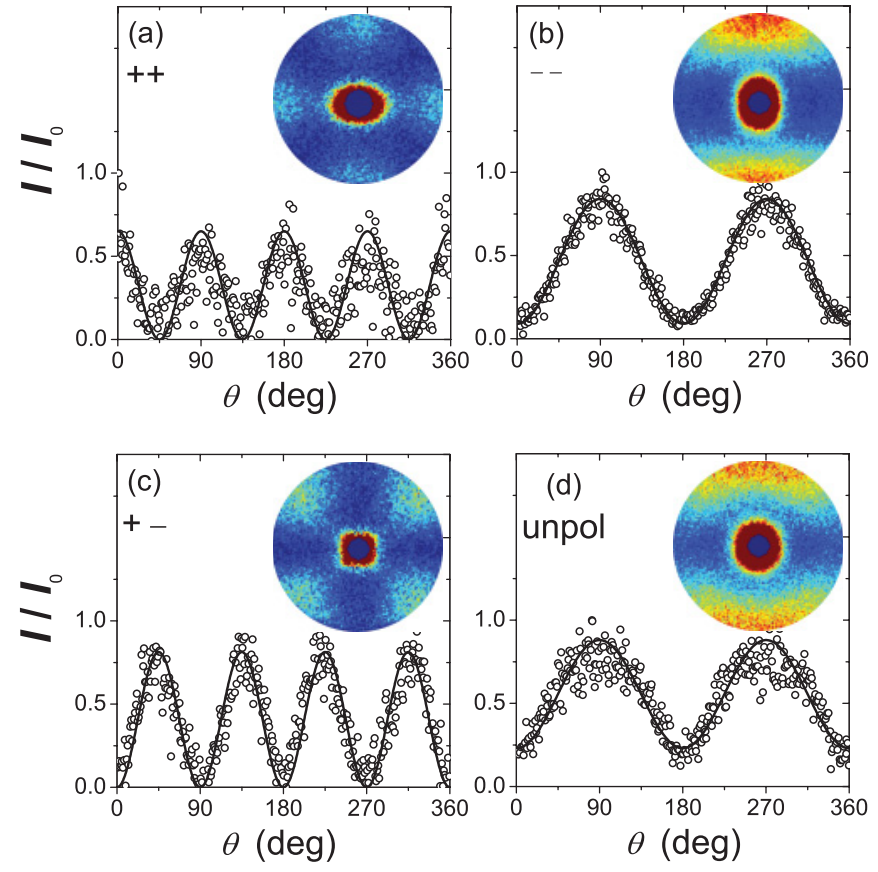

FIG. 2. (Color online) (०) Normalized intensity $I / I_{0}$ as a function of the angle $\theta$ at $\mu_{0} H=1.27 \mathrm{~T}$ and at $q=(0.26 \pm 0.01) \mathrm{nm}^{-1}$. (a) Non-spin-flip (++); (b) non-spin-flip (--); (c) spin-flip (+-), and (d) total unpolarized SANS cross section. Solid lines in (a)-(d) are guides to the eyes. Insets show the respective $\frac{d \Sigma}{d \Omega}$ on the $2 \mathrm{D}$ detector (logarithmic color scale). Applied-field direction is horizontal.

$\mu_{0} H=1.27 \mathrm{~T}$ and at $q=(0.26 \pm 0.01) \mathrm{nm}^{-1}$. Both $\frac{d \Sigma^{--}}{d \Omega}$ [Fig. 2(b)] and the total (nuclear and magnetic) unpolarized SANS cross section $\frac{d \Sigma}{d \Omega}=\frac{1}{2}\left(\frac{d \Sigma^{++}}{d \Omega}+\frac{d \Sigma^{--}}{d \Omega}+\frac{d \Sigma^{+-}}{d \Omega}+\frac{d \Sigma^{-+}}{d \Omega}\right)$ [Fig. 2(d)] show an enhanced SANS intensity normal to the field direction, in qualitative agreement with the theoretical prediction [Eqs. (3) and (4)]. The sf data at saturation [Fig. 2(c)] exhibit an anisotropy which is almost exclusively of the $\sin ^{2} \theta \cos ^{2} \theta$ type, i.e., the maxima in $\frac{d \Sigma^{+-}}{d \Omega}$ are located along the diagonals of the detector at $\theta \cong 45^{\circ}, 135^{\circ}, 225^{\circ}, 315^{\circ}$. $\frac{d \Sigma^{++}}{d \Omega}$ [Fig. 2(a)] reveals a cross-shaped angular anisotropy with maxima at $\theta \cong 0^{\circ}, 90^{\circ}, 180^{\circ}, 270^{\circ}$. By comparison to Fig. 1(b) and with reference to the previous discussion, this observation suggests $R=\frac{d \Sigma_{\text {nuc }}}{d \Omega} / \frac{d \Sigma_{\text {mag }}}{d \Omega}<1$. Clearly, this cross-shaped feature is rather unusual in magnetic SANS and we focus in the following on its analysis, which requires the separation of nuclear and magnetic scattering.

The finding that the sf scattering is predominantly of the $\sin ^{2} \theta \cos ^{2} \theta$ type with maxima along the detector diagonals [see Fig. 2(c)] strongly indicates that the function $\left|\widetilde{M}_{z}\right|^{2}$ is independent of the angle $\theta$, i.e., $\left|\widetilde{M}_{z}\right|^{2}=\left|\widetilde{M}_{z}\right|^{2}(q)$. Consequently, radially averaging of the $2 \mathrm{D}$ data shown in Fig. 2(c) provides (at saturation) the purely magnetic scattering due to longitudinal magnetization fluctuations, $\frac{d \Sigma^{+-}}{d \Omega}(q)=\frac{8 \pi^{3}}{V} \frac{1}{8} b_{H}^{2}\left|\widetilde{M}_{z}\right|^{2}(q)$. The nuclear scattering $\frac{d \Sigma_{\text {nuc }}}{d \Omega}(q)=\frac{8 \pi^{3}}{V}|\tilde{N}|^{2}(q)$ is obtained from $\left( \pm 7.5^{\circ}\right)$ sector averages of $\frac{d \Sigma^{++}}{d \Omega}$ along $\theta=0^{\circ}$. It is worth emphasizing here that the $\frac{d \Sigma_{\text {nuc }}}{d \Omega}$ that is determined in this way is essentially free of spin-misalignment scattering [compare Eq. (1)]. This is in contrast to the estimate for $\frac{d \Sigma_{\text {nuc }}}{d \Omega}$ that would be obtained by analyzing the unpolarized SANS cross section

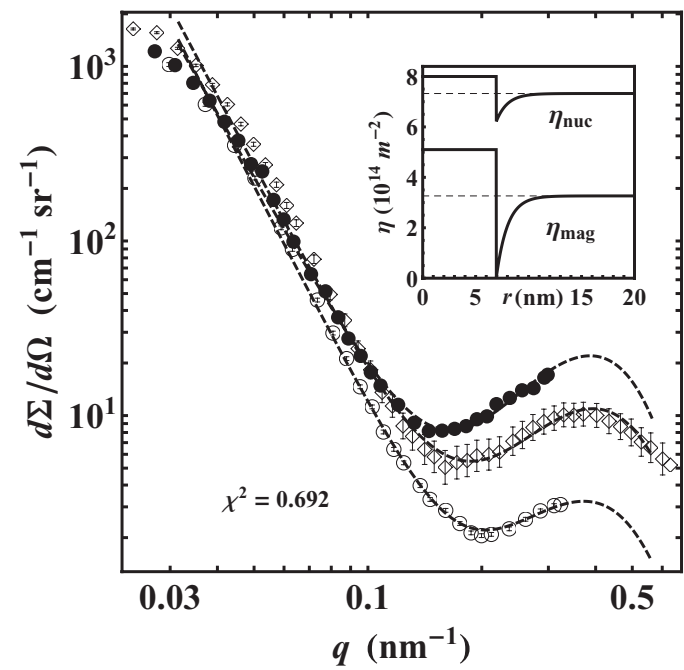

FIG. 3. Radially-averaged scattering cross sections versus momentum transfer $q$ at $\mu_{0} H=1.27 \mathrm{~T}$ (log-log scale). $(\bullet) \frac{d \Sigma_{\mathrm{mag}}}{d \Omega}=$ $\frac{8 \pi^{3}}{V} b_{H}^{2}\left|\tilde{M}_{z}\right|^{2} ;(\circ) \frac{d \Sigma_{\text {nuc }}}{d \Omega}=\frac{8 \pi^{3}}{V}|\tilde{N}|^{2} ;(\diamond)$ total unpolarized SANS cross section. Error bars of $\frac{d \Sigma_{\text {mag }}}{d \Omega}$ and $\frac{d \Sigma_{\text {nuc }}}{d \Omega}$ (not shown) are comparable to the error bars of the unpolarized data. Dashed lines: Fit to diffusion-zone model (Ref. 12) (see main text). Inset: Nuclear and magnetic scattering-length density profiles at the phase boundary between nanoparticles and matrix.

(or SANSPOL data), which contains a term $\left|\tilde{M}_{y}\right|^{2} \cos ^{2} \theta$ that -in case of incomplete saturation-may give rise to magnetic "contamination" along the $\theta=0^{\circ}$ direction. ${ }^{16,26}$ In other words, this is one example where the POLARIS method is superior over unpolarized and SANSPOL scattering techniques. The cross sections $\frac{d \Sigma_{\text {nuc }}}{d \Omega}$ and $\frac{d \Sigma_{\mathrm{mag}}}{d \Omega}=8 \frac{d \Sigma^{+-}}{d \Omega}=$ $\frac{8 \pi^{3}}{V} b_{H}^{2}\left|\widetilde{M}_{z}\right|^{2}$ are shown in Fig. 3 .

It can be seen in Fig. 3 that over the whole $q$ range studied $\frac{d \Sigma_{\mathrm{mag}}}{d \Omega}$ is larger than $\frac{d \Sigma_{\mathrm{nuc}}}{d \Omega}$ and that the ratio $R=\frac{d \Sigma_{\mathrm{nuc}}}{d \Omega} / \frac{d \Sigma_{\mathrm{mag}}}{d \Omega}<1$ approaches a constant value for $q \gtrsim 0.2 \mathrm{~nm}^{-1}$ (see Fig. 4). At small $q$, the ratio $R$ increases, in agreement with the observed transition of the pattern to an elliptically-shaped

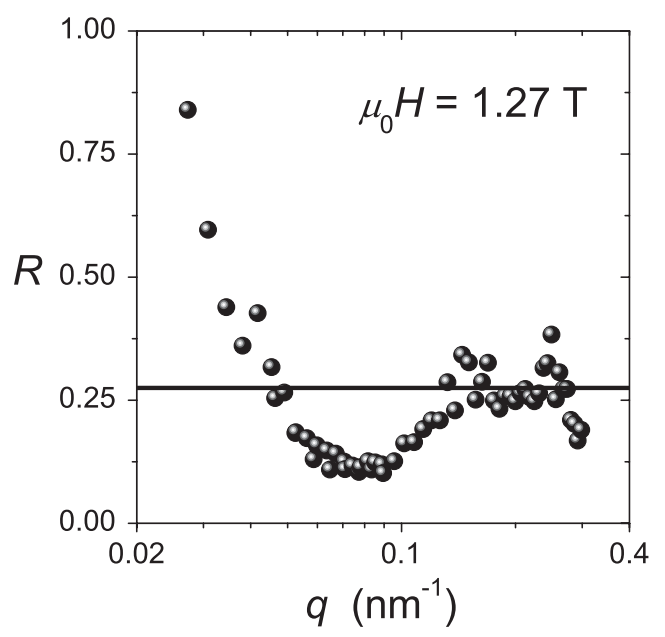

FIG. 4. Momentum-transfer dependence of the ratio $R$ of nuclear to magnetic SANS, $R=\frac{d \Sigma_{\text {nuc }}}{d \Omega} / \frac{d \Sigma_{\text {mag }}}{d \Omega}(\log$-linear scale). Horizontal line: $R=0.275$. 
anisotropy in this $q$ range with maxima along the applied-field direction [compare Figs. 1(c) and 2(a)]. ${ }^{24}$ If one assumes that the nuclear and magnetic form factors of the (spherically symmetric) nanoparticles are identical, then the value of $R$ reflects the ratio of nuclear to magnetic scattering-length density contrasts (see below). The peak in the SANS cross section at about $q_{m} \cong 0.36 \mathrm{~nm}^{-1}\left(2 \pi / q_{m} \cong 17 \mathrm{~nm}\right)$ could not be fully recorded with the POLARIS setup due to restrictions imposed by the analyzer cell.

The dashed lines in Fig. 3 represent a fit of the data to a model of polydisperse noninterfering spherical particles with diffusion zones (for details see Ref. 12). All data were fitted by means of a nonlinear fitting routine using known constraints between the curves. The larger accessible $q$ range of the unpolarized data set allowed for a significant increase of the accuracy of the fit results for the geometrical properties of the model like the mean particle diameter and the diffusionzone length. At very small $q$ values the fits differ from the data outside the range that is specified by the error bars. This observation is most likely due to the starting influence of interparticle interference effects that should be accounted for with a structure factor in the model. However, since only 3-4 data points are affected, these effects can be neglected for our purpose. From the analysis we find a mean particle size of $D_{\text {DZ }}=14 \mathrm{~nm}$ which agrees well with the finding from wideangle x-ray diffraction. The nuclear and magnetic scatteringlength density profiles at the interphase (see inset in Fig. 3) suggest the existence of a diffusion zone with a size of about $1 \mathrm{~nm}$ around the nanoparticles. ${ }^{12}$ In the following, we will discuss an alternative and complementary analysis of the data in terms of a simple two-phase model with a sharp interface.

For SANS and for a simple two-phase particle-matrix system, the magnetic scattering-length density contrast $\Delta \eta_{\text {mag }}^{2}$ between the particles ("p") and the matrix ("m") can be written as $\Delta \eta_{\text {mag }}^{2}=\left(\eta_{\text {mag }}^{\mathrm{p}}-\eta_{\text {mag }}^{\mathrm{m}}\right)^{2}=b_{H}^{2}\left(M_{s}^{\mathrm{p}}-M_{s}^{\mathrm{m}}\right)^{2}$, where $\eta_{\text {mag }}^{\mathrm{p}}$ and $\eta_{\text {mag }}^{\mathrm{m}}$ denote the magnetic scattering-length densities of the particle and matrix phase, respectively, and $M_{s}^{\mathrm{p}}$ and $M_{s}^{\mathrm{m}}$ represent the corresponding saturation-magnetization values. A rough estimate for the jump $\Delta M=M_{s}^{\mathrm{p}}-M_{s}^{\mathrm{m}}>0$ in the magnetization magnitude at the interphase boundary can be obtained via the relation $J_{s}=x_{C} \mu_{0} M_{s}^{\mathrm{p}}+\left(1-x_{C}\right) \mu_{0} M_{s}^{\mathrm{m}}$. Using $\mu_{0} M_{s}^{\mathrm{p}}=2.2 \mathrm{~T}$ (as for $\left.\alpha-\mathrm{Fe}\right), x_{C}=65 \%$, and $J_{s}=1.64 \mathrm{~T}$, we obtain $\mu_{0} M_{s}^{\mathrm{m}}=0.6 \mathrm{~T}$ for the amorphous matrix phase and, hence, $\mu_{0} \Delta M=1.6 \mathrm{~T}$ and $\Delta \eta_{\mathrm{mag}} \cong 3.7 \times 10^{14} \mathrm{~m}^{-2}$.

At large momentum transfers (and $\mu_{0} H=1.27 \mathrm{~T}$ ), the experimental value for the ratio $R$ of nuclear to magnetic scattering is $R \cong 0.275$ (compare Fig. 4 ). ${ }^{27}$ By assuming that this value for $R$ is identical to the ratio of the nuclear to magnetic scattering-length density contrasts, $R=\Delta \eta_{\text {nuc }}^{2} / \Delta \eta_{\text {mag }}^{2}$, we find for the absolute value of the nuclear scattering-length density contrast $\Delta \eta_{\text {nuc }} \cong 1.9 \times 10^{14} \mathrm{~m}^{-2}$. For $\alpha$-Fe particles, the nuclear scattering-length density equals $\eta_{\text {nuc }}^{\mathrm{p}}=8.0 \times$ $10^{14} \mathrm{~m}^{-2}$ (Ref. 28), which suggests $\eta_{\text {nuc }}^{\mathrm{m}} \cong 6.1 \times 10^{14} \mathrm{~m}^{-2}$ for the matrix (assuming also that $\eta_{\text {nuc }}^{\mathrm{p}}>\eta_{\text {nuc }}^{\mathrm{m}}$ ).

Both types of data analysis, the diffusion-zone model and the simple two-phase approach, involve more or less rough approximations to the complex nuclear and magnetic microstructure of the nanocrystalline alloy NANOPERM. The absolute values of the magnetic and nuclear contrasts which result from the diffusion-zone model, $\Delta \eta_{\text {mag }}^{\mathrm{DZ}} \cong 1.8 \times 10^{14} \mathrm{~m}^{-2}$ and $\Delta \eta_{\text {nuc }}^{\mathrm{DZ}} \cong 0.7 \times 10^{14} \mathrm{~m}^{-2}$ (compare inset in Fig. 3), yield a ratio of $R^{\mathrm{DZ}} \cong 0.15$ which is consistent with the value 0.275 found from the simple two-phase model. Hence, both analyses independently support the conclusion drawn from the experimental observation of the cross-shaped angular anisotropy in $\frac{d \Sigma^{++}}{d \Omega}$ (at saturation), i.e., the ratio of nuclear to magnetic scattering must be smaller than unity and roughly in the range between 0.1 and 0.4 .

\section{SUMMARY AND CONCLUSIONS}

At a saturating applied magnetic field of $1.27 \mathrm{~T}$, we have observed a cross-shaped angular anisotropy in the non-spinflip small-angle neutron scattering (SANS) cross section $\frac{d \Sigma^{++}}{d \Omega}$ of the Fe-based two phase nanocrystalline alloy NANOPERM. For this type of material, the cross-shaped anisotropy is only observable at saturation in $\frac{d \Sigma^{++}}{d \Omega}$, provided that the ratio of nuclear to magnetic scattering is smaller than unity. Analysis of the spin-resolved neutron data allowed us to estimate the magnitudes of the nuclear and magnetic scattering-length density contrasts at the particle-matrix interphase. Our study represents an example where spin-resolved SANS (so-called POLARIS) provides information that is not accessible via conventional SANS or SANSPOL techniques.

\section{ACKNOWLEDGMENTS}

This work was supported by the Deutsche Forschungsgemeinschaft under the Project No. MI 738/6-1 and by the National Research Fund of Luxembourg in the framework of ATTRACT Project No. FNR/A09/01. *andreas.michels@uni.lu

${ }^{1}$ G. Herzer, in Handbook of Magnetic Materials, edited by K. H. J. Buschow, Vol. 10 (Elsevier, Amsterdam, 1997), pp. 415-62.

${ }^{2}$ M. E. McHenry, M. A. Willard, and D. E. Laughlin, Prog. Mater. Sci. 44, 291 (1999).

${ }^{3}$ K. Suzuki and G. Herzer, in Advanced Magnetic Nanostructures, edited by D. Sellmyer and R. Skomski (Springer, New York, 2006), pp. $365-401$.
${ }^{4}$ J. Petzold, Scr. Mater. 48, 895 (2003).

${ }^{5}$ H. Hermann, A. Heinemann, N. Mattern, and A. Wiedenmann, Europhys. Lett. 51, 127 (2000).

${ }^{6}$ M. Ohnuma, J. Suzuki, S. Funahashi, T. Ishigaki, H. Kuwano, and Y. Hamaguchi, Mater. Trans., JIM 36, 918 (1995).

${ }^{7}$ J. Kohlbrecher, A. Wiedenmann, and H. Wollenberger, Z. Physik B 104, 1 (1997). 
${ }^{8}$ A. Danzig, A. Wiedenmann, and N. Mattern, J. Phys.: Condens. Matter 10, 5267 (1998).

${ }^{9}$ J. Marcin, A. Wiedenmann, and I. Škorvánek, Phys. B 276-278, 870 (2000).

${ }^{10}$ I. V. Golosovsky, G. Török, E. I. Maltzev, and I. Mirebeau, Phys. B 276-278, 918 (2000).

${ }^{11}$ M. Ohnuma, K. Hono, S. Linderoth, J. S. Pedersen, Y. Yoshizawa, and H. Onodera, Acta Mater. 48, 4783 (2000).

${ }^{12}$ A. Heinemann, H. Hermann, A. Wiedenmann, N. Mattern, and K. Wetzig, J. Appl. Crystallogr. 33, 1386 (2000).

${ }^{13}$ A. Michels, R. N. Viswanath, and J. Weissmüller, Europhys. Lett. 64, 43 (2003).

${ }^{14}$ A. Grob, S. Saranu, U. Herr, A. Michels, R. N. Viswanath, and J. Weissmüller, Phys. Status Solidi A 201, 3354 (2004).

${ }^{15}$ A. Michels, C. Vecchini, O. Moze, K. Suzuki, P. K. Pranzas, J. Kohlbrecher, and J. Weissmüller, Phys. Rev. B 74, 134407 (2006).

${ }^{16}$ A. Michels and J. Weissmüller, Rep. Prog. Phys. 71, 066501 (2008).

${ }^{17}$ R. M. Moon, T. Riste, and W. C. Koehler, Phys. Rev. 181, 920 (1969).

${ }^{18}$ D. Honecker, A. Ferdinand, F. Döbrich, C. D. Dewhurst, A. Wiedenmann, C. Gómez-Polo, K. Suzuki, and A. Michels, Eur. Phys. J. B 76, 209 (2010).

${ }^{19}$ K. L. Krycka, R. A. Booth, C. R. Hogg, Y. Ijiri, J. A. Borchers, W. C. Chen, S. M. Watson, M. Laver, T. R. Gentile, L. R. Dedon, S. Harris, J. J. Rhyne, and S. A. Majetich, Phys. Rev. Lett. 104, 207203 (2010).
${ }^{20}$ U. Keiderling, Appl. Phys. A 74, S1455 (2002).

${ }^{21}$ K. Suzuki, A. Makino, A. Inoue, and T. Masumoto, J. Magn. Soc. Jpn. 18, 800 (1994).

${ }^{22}$ S. Erokhin, D. Berkov, N. Gorn, and A. Michels, Phys. Rev. B 85, 024410 (2012).

${ }^{23}$ S. Erokhin, D. Berkov, N. Gorn, and A. Michels, Phys. Rev. B 85, 134418 (2012).

${ }^{24} \mathrm{We}$ note that the transition in $\frac{d \Sigma^{++}}{d \Omega}$ from the cross-shaped to a predominantly elliptic pattern sets already in for $R$ values smaller than but close to unity.

${ }^{25}$ A. Wiedenmann, Phys. B 356, 246 (2005).

${ }^{26}$ M. Bischof, P. Staron, A. Michels, P. Granitzer, K. Rumpf, H. Leitner, C. Scheu, and H. Clemens, Acta Mater. 55, 2637 (2007).

${ }^{27}$ Using the unpolarized SANS data [Fig. 2(d)], we have also computed partial Porod invariants (see Ref. 8). The ratio of the nuclear Porod invariant (determined from a sector average along $\theta=0^{\circ}$ ) to the magnetic Porod invariant (determined along $\theta=90^{\circ}$ ) equals 0.35 , which is in reasonable agreement with the asymptotic value of 0.275 , estimated from the $q$-dependent ratio of nuclear to magnetic scattering (see Fig. 4).

${ }^{28}$ The nuclear scattering-length density is computed as $\eta_{\text {nuc }}=$ $b_{\text {nuc }}^{\text {coh }} N_{A} \rho / M$, where $b_{\text {nuc }}^{\text {coh }}$ is the bound nuclear coherent scattering length (Ref. 29), $N_{A}$ is Avogadro's number, $\rho$ is mass density, and $M$ denotes the molar mass.

${ }^{29}$ V. F. Sears, Neutron News 3, 26 (1992). 